\title{
DISTURBED FLOW IN A STENOSED CAROTID ARTERY BIFURCATION: COMPARISON OF RANS-BASED TRANSITIONAL MODEL AND LES WITH EXPERIMENTAL MEASUREMENTS
}

\author{
JOHARI N.H. ${ }^{\dagger}{ }^{\dagger}$, WOOD N.B. ${ }^{*}$, CHENG Z. ${ }^{*}$, TORII R. ${ }^{*}$ \\ * Department of Chemical Engineering, Imperial College London, South Kensington, London \\ SW7 2AZ, United Kingdom \\ ${ }^{\dagger}$ Faculty of Mechanical Engineering, Universiti Malaysia Pahang, 26600 Pahang, Malaysia \\ ₹ Department of Mechanical Engineering, University College London, London WC1E 7JE, \\ United Kingdom \\ OISHI M., OSHIMA M. \\ Institute of Industrial Science, University of Tokyo, Tokyo, Japan \\ XUX.Y. \\ Department of Chemical Engineering, Imperial College London, South Kensington, London \\ SW7 2AZ, United Kingdom \\ yun.xu@imperial.ac.uk
}

Received date

Accepted date

Blood flow in the carotid arteries is usually laminar, but can undergo laminar-turbulent transition in the presence of a high-grade stenosis. In this study, pulsatile flow in a patientbased stenosed carotid artery bifurcation was examined using both large eddy simulation (LES) with dynamic Smagorinsky eddy viscosity model, and a Reynolds-averaged NavierStokes (RANS) method with a transitional version of the shear stress transport (SST-Tran) model. In addition, an experimental phantom was built for the same bifurcation geometry and velocity measurements were made using particle image velocimetry (PIV). Comparisons with PIV measurements of axial velocity profiles demonstrated that both SST-Tran and LES predicted the experimental results fairly well, with LES being slightly superior. Furthermore, LES predicted cycle-to-cycle variations in the region where transition to turbulence occurred, indicating the unsteady nature of turbulence transition. On the other hand, the SST-Tran model was able to capture important flow features observed in the PIV experiment, demonstrating its potential as a cost-effective alternative to LES for haemodynamic analyses of highly disturbed flow in diseased arteries.

Keywords: Large eddy simulation; transitional flow; particle image velocimetry; carotid artery bifurcation; stenosis. 


\section{Introduction}

Blood flow in healthy human arteries is usually laminar at rest, but transition to turbulent flow can occur in exercise and under pathological conditions, such as aortic valve stenosis or arterial stenosis. Previous experimental studies [Ahmed and Giddens, 1983, 1984; Kefayati et al., 2014] have provided evidence of laminar-turbulent transition in stenosed arteries, occurring distal to the stenosis. Transition to turbulence is also expected to be dependent on flow pulsatility and on the geometry of the stenosed artery. Previous numerical studies analysed flow in idealised straight tube stenoses [Ryval et al., 2004; Banks and Bresslo, 2007; Varghese et al., 2008; Tan et al., 2011] and patient-specific carotid bifurcations [Stroud et al., 2002; Marshall et al., 2004; Lee et al., 2008; Tan et al., 2008; Li et al., 2014, Lancellotti et al., 2017], showing the influence of the degree of narrowing on the extent of flow separation and recirculation in the post-stenotic zone.

Reynolds-averaged Navier-Stokes (RANS) turbulence models were reported to be inadequate when used to model flow through high-grade stenosis since most turbulence models cannot capture transition to turbulence [Grinberg et al., 2009; Tan et al., 2008]. Compared to RANS-based models, large eddy simulation (LES) offers an improved solution because the full unsteady Navier-Stokes equations are solved for the resolved eddies, whilst the Smagorinsky subgrid-scale approach also uses the full Navier-Stokes equations, so it displays turbulence structure. Although direct numerical simulation solves for the complete fluid flow field and can provide a wealth of detailed information on turbulence, its computational demand is prohibitive for moderate to high Reynolds number flows.

Since numerical studies differ in their solutions according to the assumptions made, a key question in computational modelling of arterial flows is validation or evaluation, especially for highly disturbed flows. Validations require well-calibrated experimental data. Computational studies including validations against experimental data have been reported on steady and pulsatile flows in a straight tube stenosis with conditions relevant to carotid stenosis [e.g. Tan et al., 2008, 2011]. More recently, transitional and turbulence flow through a simplified medical device model - the FDA benchmark nozzle model has attracted considerable attention [Taylor et al., 2016; Zmijanovic et al., 2017]. However, similar studies of more realistic arterial stenosis geometries are lacking. Therefore, the aim of this study was to evaluate the RANS SST-Tran model [Menter, 1994; Langtry, 2006] and LES with dynamic Smagorinsky [Germano et al., 1991; Lilly, 1992] by comparing computational results with the corresponding PIV measurements in a patient-based carotid stenosis model. 


\section{Methodology}

\subsection{Geometry of the Stenosed Carotid Bifurcation}

A patient-specific stenosed carotid artery bifurcation was reconstructed from magnetic resonance (MR) images, as initially reported by Tan et al., (2008). It was asymmetrical, noncylindrical and non-planar, with a $70 \%$ stenosis in the carotid bulb. The original geometry was modified in order to build a laboratory model (phantom) suitable for two-directional PIV measurement. It was achieved by removing its non-planar curvature so that the carotid bifurcation phantom lay in one plane, and the degree of stenosis was set at $80 \%$. As shown in Figure 1, the phantom was made from a block of silicone (Sylgard 184 elastomers) with a transparent wall, and its size - $18 \mathrm{~mm}$ inlet common carotid artery (CCA) diameter - was scaled up by 1.69 from the patient-specific geometry.

\subsection{Experimental Setup for PIV Measurement}

The phantom was connected to a flow generator for inflow, whilst flowmeters were installed in the outflow conduits, i.e. the external carotid artery (ECA) and internal carotid artery (ICA). A piston pump driven by a programmable waveform generator provided a physiological pulsatile flow waveform. The Coriolis digital flowmeters FD-SF8 (Keyence, Japan) were used to measure the volumetric outflow. Figure 2 shows the flow waveforms at the inlet and outlets, giving a pulse period of $1.488 \mathrm{~s}$ and an averaged ICA to ECA flow ratio of 60:40; the latter was similar to that in the original patient measured by phase contrast MRI [Tan et al., 2008]. To avoid optical distortion in the PIV measurement, the refractive index of the glycerinebased working fluid was matched to that of the silicone model, with a refractive index of 1.417 giving the best match. The working fluid has a density of $1162 \mathrm{~kg} / \mathrm{m}^{3}$ and viscosity of 6.183 mPa.s. Based on the inlet diameter of the phantom and the imposed pulsatile flow waveform, the minimum and peak Reynolds numbers were 114 and 554, respectively, and the Womersley parameter was 3.186; all representative of conditions in human carotid arteries.

For PIV acquisitions, a green double-pulse laser system (Mini-YAG 120 mJ, New Wave Research, USA) was used to provide a nominal $0.74 \mathrm{~mm}$ thick laser sheet through each of the inlet, bifurcation, and two downstream sections. The laser was synchronized with a highspeed camera (PIVCam 13-8, TSI, USA) mounted close to the test phantom. The sampling rate for the images was $7.5 \mathrm{~Hz}$ with time $500 \mu$ s between two images. 
The PIV images were processed and two in-plane velocity components were extracted at four time points for comparison with computational results. As defined in Figure 2, the first time-point (TP1) was chosen during the accelerating phase, corresponding to mid-late acceleration. The second time-point (TP2) was immediately after the peak flow. The third and fourth time points were selected to examine velocity profiles during the deceleration phase: at early flow retardation (TP3) and at mid-late retardation (TP4). Detailed comparisons were also made at three locations: location $A$ is just proximal to the carotid bifurcation, while locations $\mathrm{B}$ and $\mathrm{C}$ are distal to the stenosis.

\subsection{Computational Mesh}

The geometry used to build the carotid bifurcation phantom was exported to ANSYS ICEM CFD 15.0 (ANSYS Inc, Canonsburg, PA, USA) for mesh generation. All geometric dimensions were identical to the phantom to allow for quantitative comparisons. A multi-block structure was adopted in order to generate a structured mesh with hexahedral elements, which allows for a better control of cells especially in the region near the wall and distal to the stenosis where higher mesh density is required. Since the RANS SST-Tran and LES have different requirements for the quality and density of the computational mesh, two sets of meshes were built to satisfy their specific needs.

For simulations with SST-Tran, grid independence tests were carried out by comparing post-stenotic velocity profiles obtained with different mesh sizes. The initial mesh contained 1.4 million elements, with more elements concentrated in the stenosis area and in the immediate post-stenosis region where transition to turbulence was observed. Differences in terms of peak velocity, WSS and turbulence kinetic energy (TKE) levels were larger than 5\% between the 1.4 million elements mesh and a coarser mesh containing 970000 elements but less than $1.5 \%$ between the 1.4 million elements mesh and a finer mesh containing 2.25 million elements. Therefore, the mesh of 1.4 million hexahedral elements was chosen for the SST-Tran simulations. To ensure good grid resolution close to the vessel wall, the maximum height of wall cells in 'wall units' $(\mathrm{y}+)$ was kept at approximately 1 at the maximum, with a mean value of less than 0.4 .

For LES, the mesh density requirement is higher, since the grid size controls the amount of dissipation that represents the forward scatter of turbulent kinetic energy from the resolved scales to the subgrid scales. Moreover, implicit filtering was applied, which depends on the mesh structure [Germano et al., 1991]. In this case, further mesh refinement was performed and a mesh independence test suggested that the mesh containing 5.06 million elements 
(with $y+$ less than 1) was sufficient. Figure 3(a) shows the hexahedral elements mesh created based on an appropriate block structure in the ICA allowing for local refinement. However, the grid growth factors were kept as low as 1.2 throughout the computational domain to ensure a gradual change in element size from the core towards the wall, as shown in Figure 3(b and c).

\subsection{Flow Simulation}

Two simulation methods were adopted in this study, the RANS-based SST-Tran model [Menter, 1994; Langtry, 2006; Langtry and Menter, 2009] and LES with implicit filtering and the dynamic Smagorinsky subgrid-scale turbulence model [Germano et al., 1991; Lilly, 1992]. The SST-Tran model combines a $k$ - $\square$ model in the inner region of the boundary layer with a $k-\varepsilon$ model in the outer region and in free shear flows, together with a transitional model comprising two additional locally formulated transport equations for intermittency and the transition onset criterion in terms of the momentum thickness Reynolds number [Langtry, 2006]. It employs new correlations for transition and has been successfully applied to a wide range of engineering problems, including turbomachinery, aircraft configurations and wind turbines [Langtry and Menter, 2009]. It has also been tested for cardiovascular flow applications, showing promising results [Tan et al., 2008, 2011; Cheng et al., 2010, 2014; Kousera et al., 2012; Li et al., 2014]. The model is available in ANSYS CFX 15.0 (ANSYS Inc, Canonsburg, PA, USA). The numerical solution was obtained using an additive correction strategy, a coupled algebraic multigrid approach, giving close to second order accuracy [Hutchinson and Raithby, 1986]. A high-resolution advection scheme was adopted for spatial discretization, and a second order implicit backward Euler scheme was used for temporal discretization [Ferziger and Peric, 1986]. A fixed time step of $0.001 \mathrm{~s}$ was chosen based on previous experience with the SST-Tran model and at least three cycles were required to achieve a periodic solution.

For LES, the large scale eddies are resolved directly and the small scales are modelled using the subgrid-scale turbulence model. Smagorinsky (1963) obtained the eddy viscosity, $v_{t}$ by assuming that the small scales are in a state of equilibrium and dissipate entirely the energy received from the resolved eddies. Because there is no valid constant value of the Smagorinsky constant $\left(C_{s}\right)$, the dynamic Germano-Lilly model [Germano et al., 1991; Lilly, 1992] was used in this study (referred to here as dSm), since it allows the value for $C_{s}$ to vary over space and time depending on the instantaneous resolved flow, hence obviating the need for any ad-hoc specification of the model constant [Germano et al., 1991; Lilly, 1992]. The filter width was defined as $V^{1 / 3}$ on general grids, yielding $\left(\Delta_{x} \Delta_{y} \Delta_{z}\right)^{1 / 3}$ on Cartesian grid and the ratio of test filter width to grid filter width was about 2 [Germano et al., 1991] ( $V$ is the 
element volume; $\Delta_{x} \Delta_{y} \Delta_{z}$ are the three orthogonal dimensions of the element). The LES dSm simulations were performed with ANSYS CFX 15.0 (ANSYS Inc, Canonsburg, PA, USA). Implicit filtering, which relies on the mesh for stratifying the resolved and modelled regions, was adopted. Using a mesh with gradual expansions towards the core gives some overlap between resolved and modelled regions, with resolved eddies approaching the wall more closely than with formal filtering [Tan et al., 2011]. Central differencing scheme was used to perform spatial discretization, as is usual in LES, and the second order backward Euler scheme was used for temporal discretization [ANSYS, 2015]. Time marching was carried out with a uniform time-step of $1.0 \times 10^{-4} \mathrm{~s}$. It was found that 11 cardiac cycles were needed to ensure statistically close to convergent results and phase averages were computed for comparisons with the PIV measurements and SST-Tran simulation results.

\subsection{Boundary Conditions}

In the SST-Tran model, an inlet turbulence level was introduced to allow physiologically realistic transitional flow to occur. Turbulence intensity, Tu, can be defined as the ratio of the root mean square of the turbulent velocity fluctuations to the mean velocity, and $\mathrm{Tu}=1.5 \%$ was defined at the inlet in the SST-Tran simulations based on previous studies by Tan et al., (2008, 2011) and Li et al., (2014). For LES, a precursor initial synthetic turbulence from the SST-Tran simulation was used to initialize the LES simulation [ANSYS, 2015; Tabor and Baba-Ahmadi, 2010].

Walls were assumed to be rigid with no-slip conditions, where all velocity components were set to zero. The fluid was assumed to be Newtonian with the same kinematic viscosity and density as the working fluid used in the PIV experiment. Fully developed velocity profiles based on the ICA waveform applied in the PIV experiment were imposed at the CCA inlet, with the profiles corresponding to the same Womersley parameter as in the experiment. At the ICA outlet, a pulsatile fractional mass flow rate based on the inlet was imposed and constant zero relative pressure was defined at the ECA outlet, setting the average flow ratio of ICA to ECA at $60: 40$.

\section{Results}

\subsection{Comparison of Velocity Profiles}

Velocity profiles obtained using SST-Tran and LES dSm models were compared with the corresponding PIV measurements. First, qualitative comparisons were made in terms of axial velocity contours and velocity vectors on the central-plane as shown in Figure 4 at the 
bifurcation and Figure 5 in the post-stenotic region. In general, a clear high-velocity jet can be seen from the throat (Figure 4), which extended into the post-stenotic region along the upper wall in the ICA. Behind the stenosis, there was a large flow recirculation zone where velocities were low in magnitude (Figure 5). On the other hand, flow in the ECA was well organized and undisturbed at all time-points. These features were well captured by both computational models.

Figures 6 and 7 show detailed comparisons of velocity profiles in the axial direction $(u)$ and in the vertical direction $(v)$ at three locations and four different time-points. Generally, the predicted axial velocities were well within the standard deviations at all time-points. However, the much smaller secondary velocity component showed some deviations from the experimental results especially in the bifurcation region. Comparisons of velocity profiles obtained with LES and SST-Tran showed that LES better captured the complex flow pattern in the low velocity region, as revealed in the PIV measurements at TP2-TP4. This was likely the effect of the resolved part of the flow, reflecting the fact that turbulent flow is inherently unsteady and inhomogeneous, but the subgrid-scale treatment also gives more complex patterns. This is especially noticeable in pulsatile flow, since the flow at each time point is transitory, making the turbulence more unsteady than in a steady mean flow. This is an important factor when considering turbulence in unsteady mean flows, such as the pulsatile flow described here.

Closer comparisons between the PIV and computational results were made. At location A (Figure 6), the $u$ velocity profiles were very well predicted by both simulations, exhibiting good agreement with the PIV measurements. Differences in the peak $u$ velocity at this location were less than $10 \%$. It was also clear that both LES and SST-Tran replicated the shape of the $u$ velocity profile with a small dent near the centre, reflecting the influence of the flow divider. The $v$ velocities measured by PIV were higher than those obtained with both simulations at all time-points, although the relative uncertainty in $v$ was larger than $u$, owing to its smaller values. Both simulations consistently under-predicted the v velocity values.

At location B (Figure 7), both simulations produced similar patterns to the PIV measurements, but LES results were in much better agreement than SST-Tran results at TP2-TP4. LES clearly captured the flow feature that was observed in the PIV measurements especially in the separation zone along the lower wall, whereas the SST-Tran model underestimated the extent of flow separation at this location. The simulated velocity profiles at location $C$ had similar patterns to the PIV measurements, except that the exact position where the maximum velocity was found differed slightly. 


\subsection{Quantification of Turbulent Flow Features}

Turbulent kinetic energy (TKE) was evaluated and compared between the LES and SSTTran simulation results, although no PIV experimental data were available for comparison. TKE was calculated according to

$$
T K E=\frac{1}{2}\left(u^{\prime 2}+v^{\prime 2}+w^{\prime 2}\right)
$$

where $u^{\prime}, v^{\prime}$ and $w^{\prime}$ are the fluctuating velocity components. Using Reynolds decomposition, the fluctuating component $u$ ' can be obtained as the difference between the instantaneous velocity $u_{i}$ and the ensemble average velocity $u$. The root-mean-square of the difference between instantaneous velocity and mean velocity was calculated as [Lantz et al., 2012; Versteeg and Malalasekera, 2007]

$$
u^{\prime}=\sqrt{\frac{1}{N} \sum_{i=1}^{N}\left(u_{i}-\bar{u}\right)^{2}}
$$

where $\mathrm{N}$ corresponds to the number of pulsatile cycles.

Figure 8 shows the evolution of TKE profiles at six different locations in the post-stenotic region. The presence of laminar-turbulent transition in the ICA was captured by both simulation methods, progressing towards re-laminarization farther downstream from the stenosis. High TKE values were found at locations $Z_{1}$ and $Z_{2}$ at peak systole and during flow deceleration phase (TP3 and TP4). The TKE levels gradually decreased farther downstream as the flow left the influence of the stenosis, allowing re-laminarization as the viscous forces took effect.

In Figures 9 and 10, the vorticity magnitude and eddy-viscosity obtained with LES allow comparisons between the complementary flow fields for the two regions, resolved and modelled, in the post-stenotic ICA. Two additional time-points during deceleration phase were used for a better visualisation of the progression of post-stenotic flow. The vorticity magnitude contours (Figure 9) clearly show transition to turbulence beginning at locations 3 4 and re-laminarization beginning around locations 6 and 7 at time-points (b) - (d). Re- 
laminarization obviously began closer to the stenosis at time-points (e) - (f). The turbulent structure predicted by LES is displayed through eddy viscosity distribution. Figure 10 shows results in the range $v_{t} / v=0-0.10$, so at these low values of Reynolds number, eddy viscosity was an order of magnitude lower than fluid viscosity. During acceleration (time-point (a)), turbulence was limited to the separated jet at the upper wall of the post-stenotic area. However, beginning at peak systole, turbulence occurred in the separated zone and at the lower wall. At time-point (e) (late retardation), turbulence showed signs of re-laminarization near location 4. However, turbulent eddy viscosity was seen throughout the downstream region closer to the walls at time-point (d) which is consistent with the velocity profiles in Figures 6 and 7.

\section{Discussion}

This study focuses on the evaluation of two different simulation methodologies against PIV velocity measurements of laminar-turbulent transitional flow in a laboratory model of a severely stenosed (80\%) carotid artery bifurcation. However, it should be noted that the PIV data used for comparisons here were not instantaneous values, nor were there any turbulence data. The results represented phase data which were ensemble averaged over the last five pulsatile cycles. The combination of experimental unsteadiness and measurement uncertainty resulted in the standard deviations of velocity magnitude shown in Figures 6 and 7. SST-Tran results were repeatable after three cycles; hence results obtained in the third cycle were used for comparison. LES results were still varying after several cycles, thought to represent the genuine fluctuations of the larger eddies. The LES results were presented based on phase averaging of 11 pulsatile cycles to obtain results that were statistically close to convergence. In case the mean flow is steady, simple arithmetic average produces a representative mean turbulent velocity profile [Stein and Sabbah, 1976]. However, for pulsatile flows it is not known how many cycles would be required to obtain a true average; since the turbulence is renewed in each cycle it seems highly likely that it is impossible, although averaging a large number of cycles might provide an acceptable result.

In the stenosed carotid bifurcation model studied here, the high-degree stenosis in the ICA created a jet flow skewed to the upper wall and at a sufficiently high instantaneous value of the near-peak Reynolds number ( $R e=2255$ measured based on peak velocity at Location B), it became unstable leading to the onset of turbulence. The results agreed well with Varghese et al., (2007), Li et al. (2014) and Lancellotti et al., (2017). As the flow left the influence of the stenosis, it re-laminarised. Generally, velocities predicted by LES were in better agreement with PIV than SST-Tran at all locations, although both simulations predicted almost similar profiles in the bifurcation region. In the post-stenotic region, the 
velocity profiles computed by LES were unsteady and fluctuated during the acceleration and deceleration phases, representing the unsteady nature of turbulence, with a longer period than the pulsatile flow period. This feature cannot be captured by a RANS turbulence model. Therefore, a clearer representation of transition was expected in the LES results. The deep separation zone below the jet along the lower wall of the ICA was shown by LES to become more unsteady, with more obvious large turbulent eddies through the retardation period as the width of the main flow increased when the re-attachment region was approached. The measured velocity profiles (Figure 7) showed a large separation zone at location B, diminishing with retardation and distance downstream (location $\mathrm{C}$ ). The high standard deviations in the measurements might partly reflect the high fluctuation levels, as simulated by LES (Figure 6).

In the post-stenotic region at location B both LES and SST-Tran models slightly over or under-predicted the peak velocity recorded by the PIV, but the main differences were in the velocities in the separation zone (Figures 6 - 7). However, the broad range of experimental uncertainty in the PIV measurement is noticeable. The results in this area indicate the deep separation zone originating from the stenosis. High velocity fluctuation levels were observed at location C, especially at TP2-TP4 when the flow was predicted to be turbulent, with LES providing the turbulent flow structure.

TKE was used to quantify the turbulence levels predicted by the SST-Tran and LES models (Figure 8). The energy in turbulent flows and anisotropy effects are predominantly present in large-scale motion. The LES TKE profiles peaked within the jet-like flow along the upper wall before it scattered downstream of location $Z_{3}$, whereas SST-Tran showed a more distributed peak, perhaps influenced by the shear-layer treatment in the transitional model. The LES results at locations $Z_{1}$ and $Z_{2}$ indicated that TKE occurred in early systole with similar profiles to those at late systole, although they did not fall as the stabilising effects of acceleration progressed. The TKE profiles at location $Z_{3}$, lower than those at $Z_{1}$ and $Z_{2}$, confirmed the progress of re-laminarization throughout the time phases. Similar phenomena were measured by Stein and Sabbah (1976) using hot film sensors in human aortas at high Reynolds numbers, distal to both normal and diseased aortic valves.

It is possible to derive vorticity from SST-Tran, but without sufficient details [Tan et al., 2011]. Thus, turbulent flow features were shown more clearly by the LES vorticity contours (Figure 10). The range of relative eddy viscosity contours, $v_{t} / v$ in Figure 10 was between 0 and 0.1 , which was an order of magnitude lower than the fluid viscosity. From the relative eddy viscosity distribution (Figure 10), there was evidence of turbulence farther downstream than in the resolved turbulence, farther from the walls. It should be noted that the implicit 
filtering used in the LES simulations, influenced by the mesh structure, provides some overlap between the resolved and modelled regions [Tan et al., 2011].

Similar flow patterns have been reported in several other related numerical studies on stenosed carotid artery bifurcation models. Tan et al., (2008) presented time-averaged wall shear stress contours and turbulence intensity levels of a patient-specific carotid artery, on which the present experimental model was based. During both peak systole and middeceleration phases, SST-Tran predicted blood flow that was concentrated along the inner wall of the stenosed ICA with a low shear stress near the outer wall as a result of the separated flow. Earlier studies compared MRI velocity measurements with CFD and observed similarities in the velocity profile in both MRI and CFD [Botnar et al., 2000; Marshall et al. 2004]. Similar velocity profiles were also obtained by Li et al., (2014) in a highly stenotic patient-specific carotid artery bifurcation by combining in vivo MRI with CFD. The PIV experimental study of Kefayati et al., (2014) (with 70\% stenosis) also confirmed the presence of separated flow adjacent to the jet in the post-stenotic zone.

In this study we have provided qualitative and quantitative comparisons between PIV measurements and numerical predictions of complex flow in carotid stenosis. In particular, the new results presented here have provided further evaluation of SST-Tran for pulsatile flow in an anatomically realistic model of carotid bifurcation stenosis. It is worth commenting that in the context of CFD applications, existing uncertainties and difficulties remain in the modelling of embedded transition to turbulent flow. Arterial flows in healthy vessels are predominantly laminar, whilst there are pathological situations, mainly in the aorta and carotid arteries where laminar-turbulent transition may occur. In a dissected aorta, the flow is likely to be highly disturbed and may become transitional and turbulent in the jet through the tear and in the coarctation in the true lumen [Cheng et al., 2010]. By applying SST-Tran to a patientspecific model of aortic dissection, Cheng et al., (2014) compared their computational results with velocities measured in vivo using phase-contrast MRI. In another study of a thoracic aortic aneurysm [Tan et al., 2009], velocity profiles obtained with SST-Tran were found to agree well with the corresponding MRI data. However, direct comparison of SST-Tran with LES and experimental data for pulsatile flow in a severely stenosed carotid bifurcation was not available in the literature before the present study.

LES is computationally expensive compared with RANS-based models; however, it is being used increasingly in industrial flows, and as desktop computers become more powerful, it can more readily be used in haemodynamic applications. Previous studies investigated LES with different subgrid scale models in capturing flow structures in post-stenotic region in a straight tube at conditions representative of the internal carotid artery [Tan et al., 2011; 
Varghese et al., 2008; Pal et al., 2014] and recently in a realistic stenotic carotid artery bifurcation [Lancellotti et al., 2017].

The comparison between the PIV measurement and numerical results presented here has a number of limitations. First, the stenotic carotid bifurcation model employed in this study was planar to suit the experimental setup, whereas realistic carotid arteries have more complex geometric features. Second, only two velocity components were acquired with PIV and instantaneous measurements were not available for comparison. Finally, the PIV measurement had limited spatial resolution and did not allow for an accurate superimposition between the experimental and computational results.

\section{Conclusion}

In this study, flow analysis was carried out for a patient-based PIV model of a stenosed carotid artery bifurcation using two different turbulence simulation methodologies, RANS SST-Tran and LES with dynamic Smagorinsky. Comparisons with PIV measurements in terms of axial velocity profiles demonstrated that both SST-Tran and LES predicted the experimental results reasonably well, with LES being slightly superior. This study also revealed the capability of both turbulence models in capturing the complex post-stenotic flow patterns.

LES with dynamic Smagorinsky model produced the best overall agreement with the experimental data throughout the pulsatile cycle with the inflow Reynolds number varying between 114 and 554, demonstrating the suitability of the modelling approach for physiologically relevant, low-Reynolds number transitional flow. The effect of $80 \%$ stenosis was well-captured by the LES where the transition to turbulence was expected to occur especially when the Reynolds number at the stenosis exceeded 2000. The velocity profiles predicted by the LES in the post-stenotic region exhibited cycle-to-cycle variations, before the flow relaminarized as it escaped the influence of the stenosis, indicating the unsteady nature of turbulence transition. However, LES simulation is computationally costly, especially for pulsatile flow in complex patient-specific geometries. On the other hand, the RANS SST-Tran model also managed to capture important flow features, i.e. flow separation and reattachment as observed in the experiment. The velocity profiles were almost identical to those obtained with LES at the bifurcation region but differed slightly distal to the stenosis. The comparisons gave credence to the RANS-based SST-Tran model in that the comprehensive engineering correlations incorporated in the model could be applied to lower Reynolds number transition to turbulent flow with some reservations regarding the onset of turbulence. Together with previous studies of pulsatile flow in a severely stenosed patient- 
specific carotid bifurcation [Li et al., 2014], disturbed flow in realistic aorta [Kousera et al., 2013] and aortic dissections [Cheng et al., 2010, 2014], this study reiterates the potential suitability of the transitional model as a cost-effective alternative to LES, for haemodynamic analyses of diseased arteries.

\section{References}

Ahmed, S. A. and Giddens, D. P. [1983] "Flow Disturbance Measurements through a Constricted Tube at Moderate Reynolds Numbers," Journal of Biomechanics 16(12), 955963.

Ahmed, S. A. and Giddens, D. P. [1984] "Pulsatile Poststenotic Flow Studies with Laser Doppler Anemometry," Journal of Biomechanics 17(9), 695-705.

ANSYS [2015]. ANSYS CFX-Solver Modeling Guide, (ANSYS Inc., Canonsburg, PA)

Banks, J. and Bresslo N. W. [2007] "Turbulence Modeling in Three-Dimensional Stenosed Arterial Bifurcations," Journal of Biomechanical Engineering 129(1): 40-50.

Botnar, H., Rappitsch, G., Scheidegger, M. B., Liepsch, D., Perktold, K. and Boe-siger, P. [2000] "Hemodynamics in the Carotid Artery Bifurcation: A Comparison between Numerical Simulations and In Vitro MRI Measurements," Journal of Biomechanics 33(2), 137-144.

Cheng, Z., Juli, C., Wood, N. B., Gibbs, R. G. J. and Xu, X. Y. [2014] "Predicting Flow in Aortic Dissection: Comparison of Computational Model with PC-MRI Velocity Measurements," Medical Engineering and Physics 36(9), 1176-1184.

Cheng, Z., Tan, F. P. P., Riga, C. V., Bicknell, C. D., Hamady, M. S., Gibbs, R. G. J., Wood, N. B. and $\mathrm{Xu}, \mathrm{X}$. Y. [2010] "Analysis of Flow Patterns in a Patient-Specific Aortic Dissection Model," Journal of Biomechanical Engineering 132(5), 51007.

Ferziger, J. H. and Peric, M. [1996] Computational Methods for Fluid Dynamics (Springer, Berlin).

Germano, M., Piomelli, U., Moin, P. and Cabot, W. H. [1991] “A Dynamic Subgrid-Scale Eddy Viscosity Model," Physics of Fluids A-Fluid Dynamics 3(7), 1760-1765.

Grinberg, L., Yakhot, A. and Karniadakis, G. E. [2009] "Analyzing Transient Turbulence in a Stenosed Carotid Artery by Proper Orthogonal Decomposition," Annals of Biomedical Engineering 37(11), 2200-2217.

Hutchinson, B. R. and Raithby, G. D. [1986] "A Multigrid Method Based on the Additive Correction Strategy," Numerical Heat Transfer 9(5): 511-537. 
Kefayati, S., Holdsworth, D. W. and Poepping, T. L. [2014] "Turbulence Intensity Measurements Using Particle Image Velocimetry in Diseased Carotid Artery Models: Effect of Stenosis Severity, Plaque Eccentricity, and Ulceration," Journal of Biomechanics 47(1): 253-263.

Kousera, C. A., Wood, N. B., Seed, W. A., Torii, R., O'Regan, D. and Xu, X. Y. [2012] "A Numerical Study of Aortic Flow Stability and Comparison With In Vivo Flow Measurements," Journal of Biomechanical Engineering 135(1), 11003.

Lancellotti, R. M., Vergara, C., Valdettaro, L., Bose, S. and Quarteroni, A. [2017] "Large Eddy Simulation for Blood Dynamics in Realistic Stenotic Carotids," International Journal for Numerical Methods in Biomedical Engineering 33(11), 1-14.

Langtry, R. B., Menter, F. R., Likki, S. R., Suzen, Y. B., Huang, P. G. and Volker, S. [2006] "A Correlation-based Transition Model using Local Variables Part II: Test Cases and Industrial Applications," Journal of Turbomachinery 128(3), 423-434.

Langtry, R. B. and Menter. F. R. [2009] "Correlation-Based Transition Modeling for Unstructured Parallelized Computational Fluid Dynamics Codes," AIAA Journal 47(12): 2894-2906.

Lantz, J., Ebbers, T., Engvall, J. and Karlsson, M. [2013] "Numerical and Experimental Assessment of Turbulent Kinetic Energy in an Aortic Coarctation," Journal of Biomechanics 46(11), 1851-1858.

Lee, S. E., Lee, S. W., Fischer, P. F., Bassiouny, H. S. and Loth, F. [2008] "Direct Numerical Simulation of Transitional Flow in a Stenosed Carotid Bifurcation," Journal of Biomechanics 41(11), 2551-2561.

Lee, S. W., Antiga, L., Spence, J. D. and Steinman, D. A. [2008] "Geometry of the Carotid Bifurcation Predicts Its Exposure to Disturbed Flow," Stroke 39(8): 2341-2348.

Li, Z. Y., Tan, F. P. P., Soloperto, G., Wood, N. B., Xu, X. Y. and Gillard, J. H. [2014] "Flow Pattern Analysis in a Highly Stenotic Patient-specific Carotid Bifurcation Model using a Turbulence Model," Computer Methods in Biomechanics and Biomedical Engineering 18(10), 1099-1107.

Lilly, D. K. [1992] "A Proposed Modification of the Germano-Subgrid-Scale Closure Method," Physics of Fluids A-Fluid Dynamics 4(3), 633-635.

Marshall, I., Zhao, S., Papathanasopoulou, P., Hoskins, P. and Xu, X. Y. [2004] "MRI and CFD Studies of Pulsatile Flow in Healthy and Stenosed Carotid Bifurcation Models," Journal of Biomechanics 37(5), 679-687.

Menter, F. R. [1994] "Two-Equation Eddy-Viscosity Turbulence Models for Engineering Applications," AIAA Journal 32(8), 1598-1605.

Pal, A., Anupindi, K., Delorme, Y. T., Ghaisas, N., Shetty, D. A. and Frankel, S. H. [2014] "Large Eddy Simulation of Transitional Flow in an Idealized Stenotic Blood Vessel: Evaluation of Subgrid Scale Models," Journal of Biomechanical Engineering 136(7), 1-8. 
Ryval, J., Straatman, A. G. and Steinman, D. A. [2004] "Two-Equation Turbulence Modeling of Pulsatile Flow in a Stenosed Tube," Journal of Biomechanical Engineering 126(5), 625635.

Schirmer, C. M. and Malek, A. M. [2012] "Computational Fluid Dynamic Characterization of Carotid Bifurcation Stenosis in Patient-Based Geometries," Brain and Behavior 2(1), 42-52. Smagorinsky, J. [1963] "General Circulation Experiments with the Primitive Equations," Monthly Weather Review 91(3), 99-164.

Stein, P. D. and Sabbah, H. N. [1976] "Turbulent Blood Flow in the Ascending Aorta of Humans with Normal and Diseased Aortic Valves," Circulation Research 39(1), 58-65.

Stroud, J. S., Berger, S. A. and Saloner, D. [2002] "Numerical Analysis of Flow through a

Severely Stenotic Carotid Artery Bifurcation," Journal of Biomechanical Engineering 124(1), 9-20.

Tabor, G. and Baba-Ahmadi, M. H. [2010]. "Inlet Conditions for Large Eddy Simulation: A Review," Computers and Fluids 39(4), 553-567.

Tan, F. P. P., Soloperto, G., Bashford, S., Wood, N. B., Thom, S., Hughes, A. and Xu, X. Y. [2008] "Analysis of Flow Disturbance in a Stenosed Carotid Artery Bifurcation using TwoEquation Transitional and Turbulence," Journal of Biomechanical Engineering 130(6), 061008.

Tan, F. P. P., Torii, R., Borghi, A., Mohiaddin, R. H., Wood, N. B. and Xu, X. Y. [2009] "FluidStructure Interaction Analysis of Wall Stress and Flow Patterns in a Thoracic Aortic Aneurysm," International Journal of Applied Mechanics 1(1), 179-199.

Tan, F. P. P., Wood, N. B., Tabor, G. and Xu, X. Y. [2011] "Comparison of LES of Steady Transitional Flow in an Idealized Stenosed Axisymmetric Artery Model with a RANS Transitional," Journal of Biomechanical Engineering 133(5), 051001.

Taylor, J. O., Good, B. C., Paterno, A. V., Hariharan, P, Deutsch, S., Malinauskas, R. A. and Manning, K. B. [2016] "Analysis of Transitional and Turbulent Flow Through the FDA Benchmark Nozzle Model Using Laser Doppler Velocimetry," Cardiovascular Engineering and Technology 7(3), 191-209.

Varghese, S. S., Frankel, S. H. and Fischer, P. F. [2007] "Direct Numerical Simulation of Stenotic Flows, Part 2: Pulsatile Flow," Journal of Fluid Mechanics 582, 281-318.

Varghese, S. S., Frankel, S. H. and Fischer, P. F. [2008] "Modeling Transition to Turbulence in Eccentric Stenotic Flows," Journal of Biomechanical Engineering 130(1), 014503.

Versteeg, H. K. and Malalasekera, W. [2007] Introduction to Computational Fluid Dynamics: The Finite Volume Method (Pearson Prentice Hall, London).

Zmijanovic, V., Mendez, S., Moureau, V. and Nicoud, F. [2016] "About the Numerical Robustness of Biomedical Benchmark Cases: Interlaboratory FDA's Idealized Medical Device," International Journal for Numerical Methods in Biomedical Engineering 33(1), e02789. 


\section{Acknowledgements}

Nasrul Johari was supported by a PhD scholarship awarded by the Government of Malaysia. The authors are grateful to Dr Felicia Tan who helped design the geometry of the carotid bifurcation phantom adopted in this study when she was a $\mathrm{PhD}$ student at Imperial College London. Support by the Royal Society International Joint Project award is also gratefully acknowledged (2007/R3/lJP). 


\section{Illustrations and Photographs}

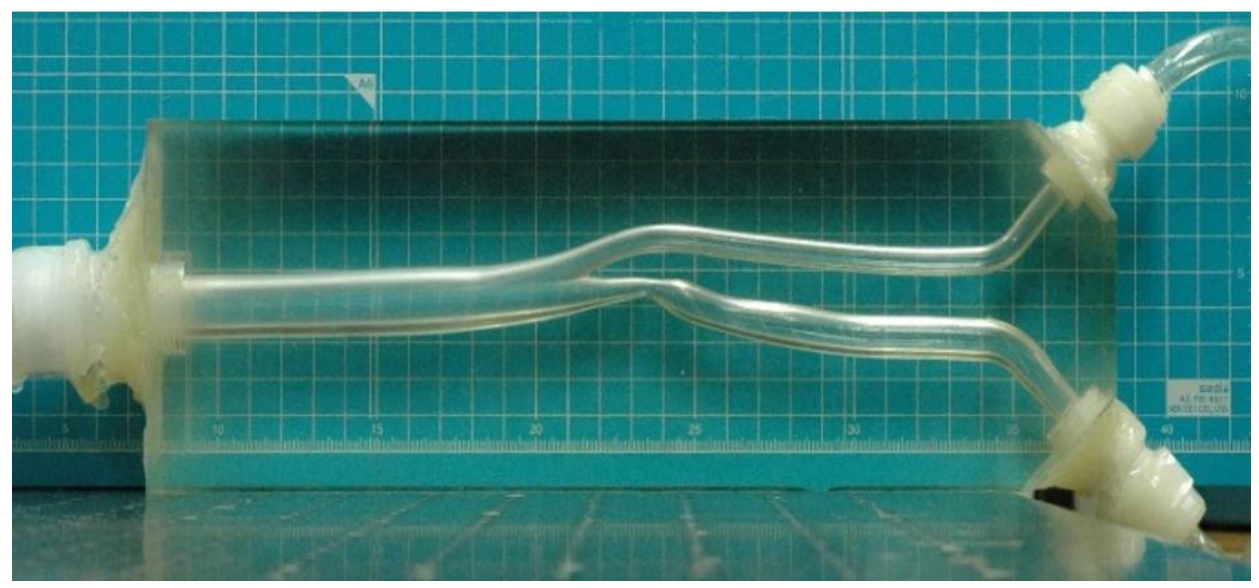

Fig. 1. Modified patient-based stenosed carotid bifurcation model for computational simulations and PIV experiment. The phantom was cast in transparent silicone within a solid block. 


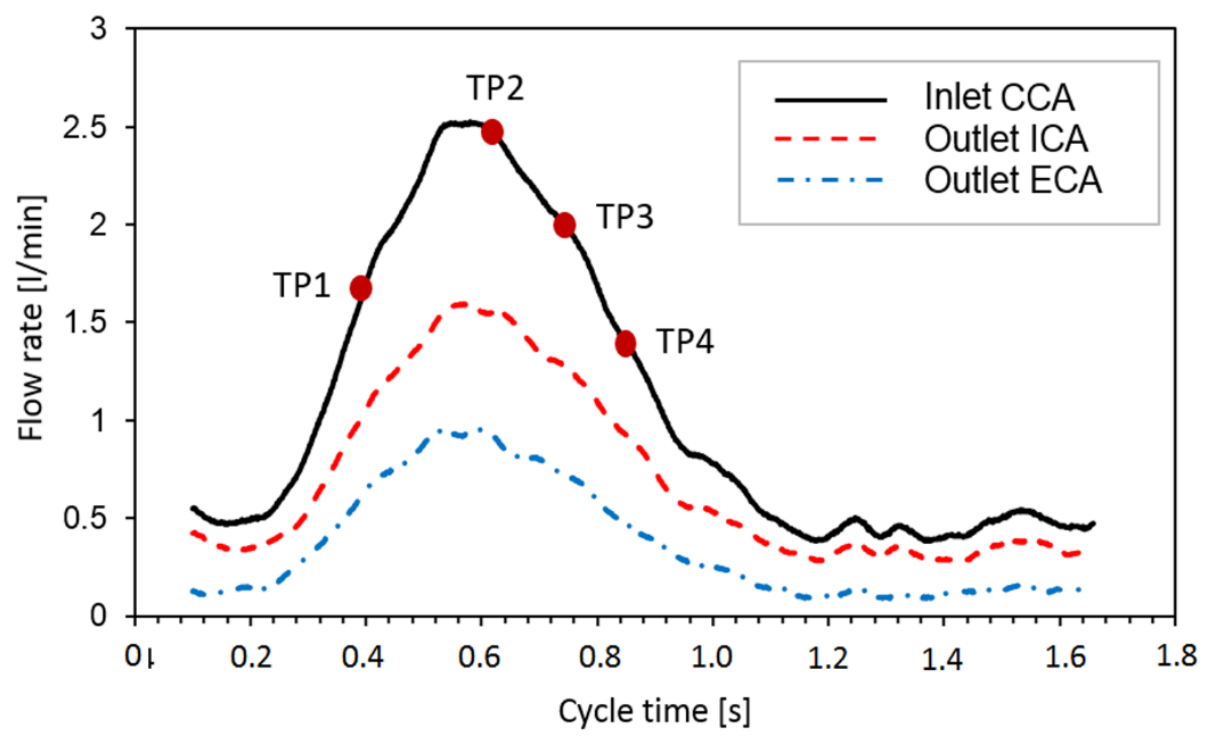

ECA

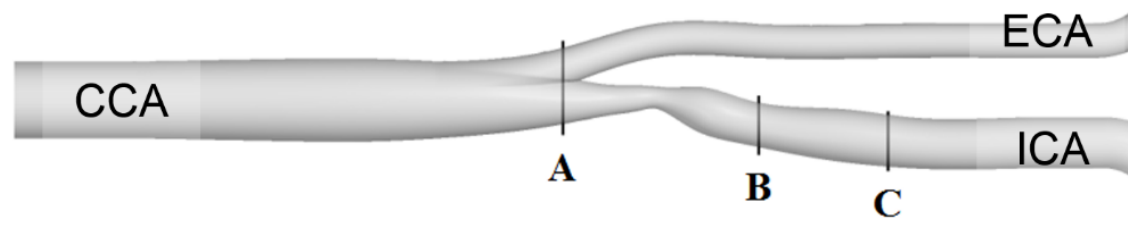

Fig. 2. Inlet and outlet flow rate waveforms in the flow rig used for PIV measurements. Velocity profiles were compared at the four selected time points and three different locations. Common carotid artery (CCA), internal carotid artery (ICA), external carotid artery (ECA) and time-point (TP). 


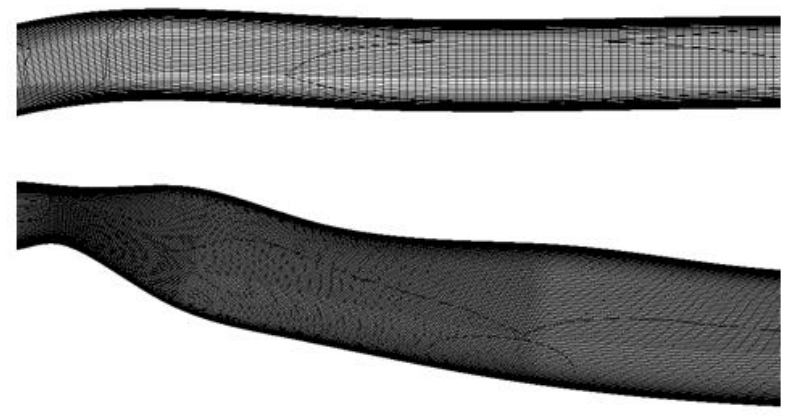

(a)
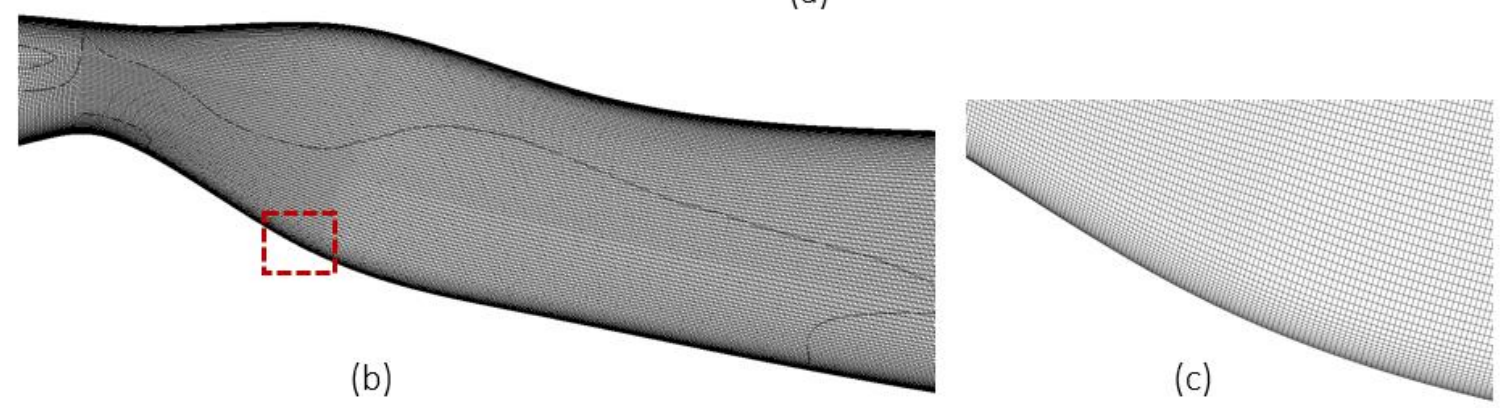

Fig. 3. The hexahedral elements mesh used in LES simulations in streamwise mid-plane view. (a): Different mesh densities used in ICA and ECA; (b): higher mesh density in the stenosis and distal stenosis area; and (c) close-up of the near wall mesh in the immediate post-stenosis area. 


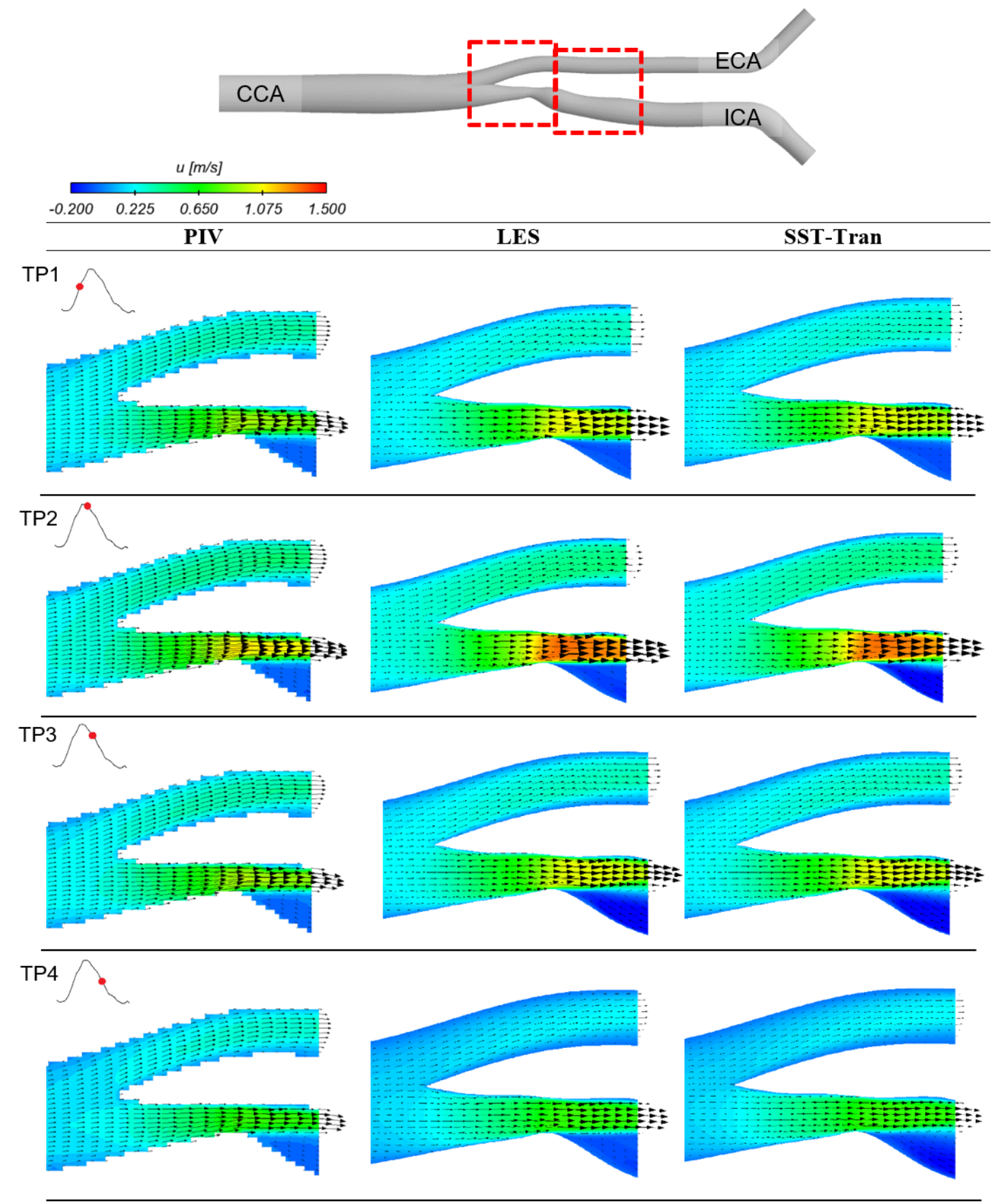

Fig. 4. Comparisons between the PIV measurements and computational results: axial velocity contours and velocity vectors in the bifurcation and stenosis region at different time-points (TP1-TP4). Results are shown in the $x-y$ plane. 


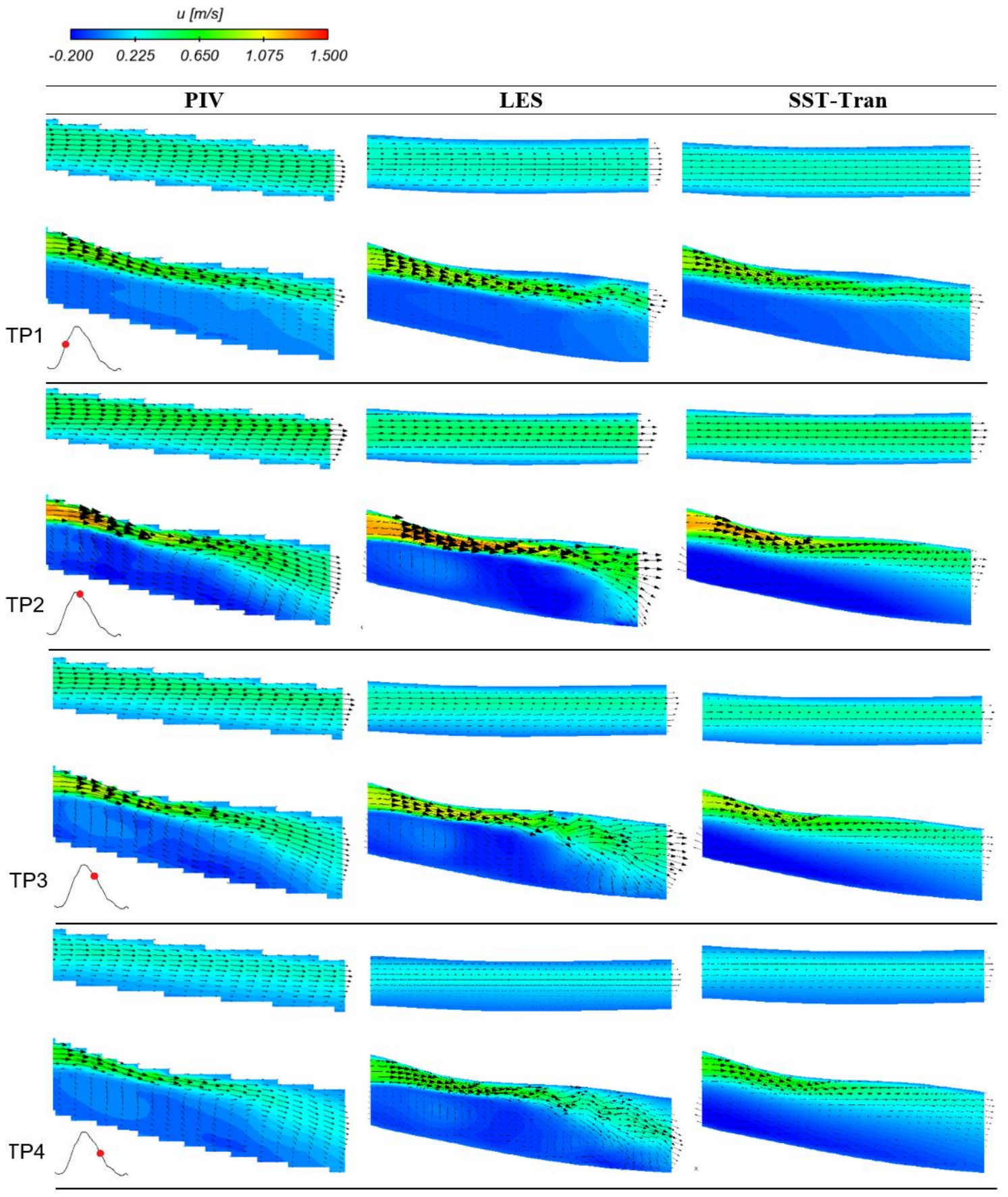

Fig. 5. Comparisons between the PIV measurements and computational results: axial velocity contours and velocity vectors in the post-stenotic area at different time-points (TP1-TP4). Results are shown in the $x-y$ plane. 
A
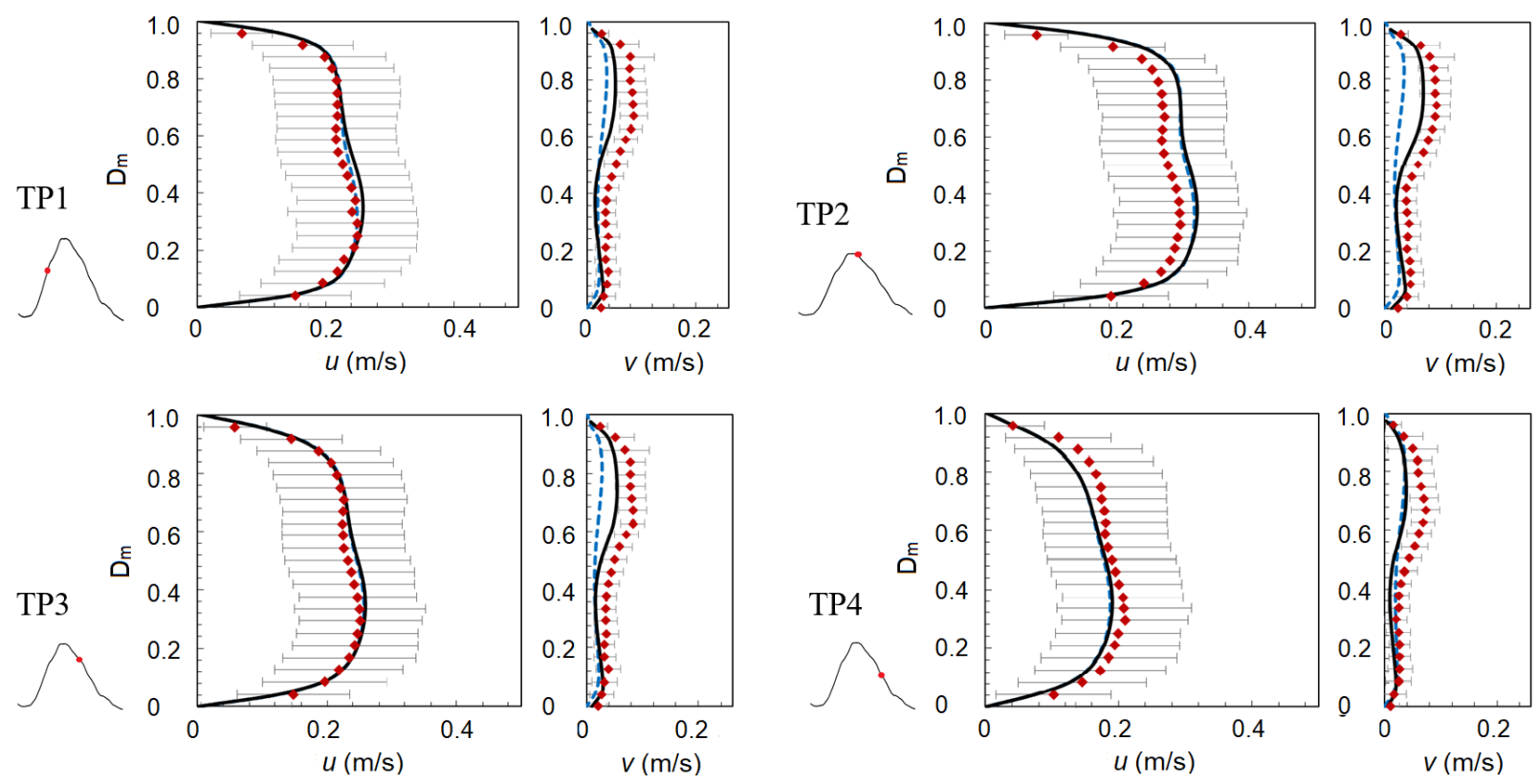

Fig. 6. Comparisons of $u$ and $v$ velocity components at a location before the bifurcation

(Location A at time points TP1-TP4. Red diamond dotted curves represent the PIV measurement, showing mean SD; blue dashed curves show SST-Tran results and black continuous curves show LES results. 

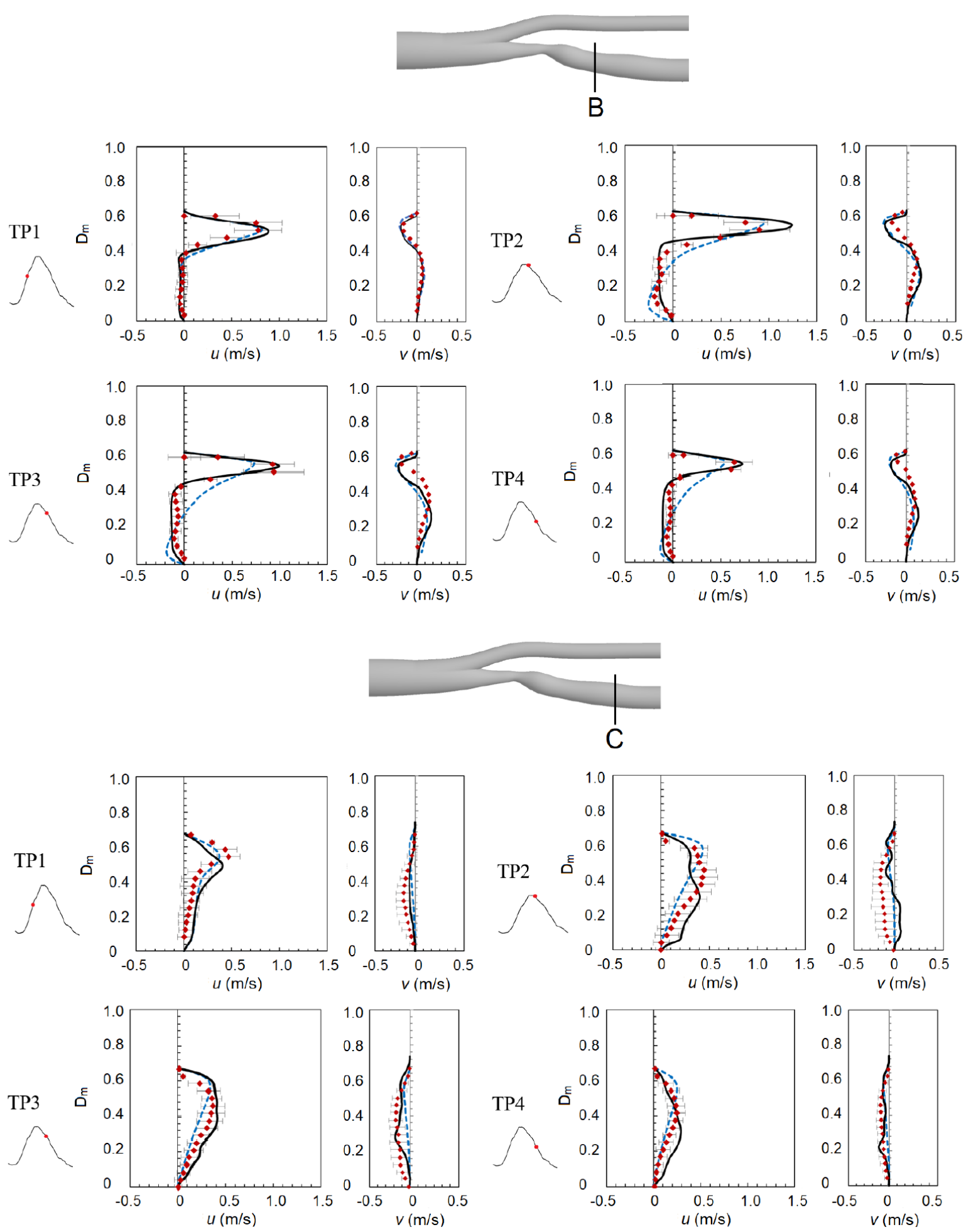

Fig. 7. Comparisons of $u$ and $v$ velocity components at locations downstream from the stenosis (Location B and C) at time points TP1-TP4. Red diamond dotted curves represent the PIV measurement, showing mean SD; blue dashed curves show SST-Tran results and black continuous curves show LES results. 

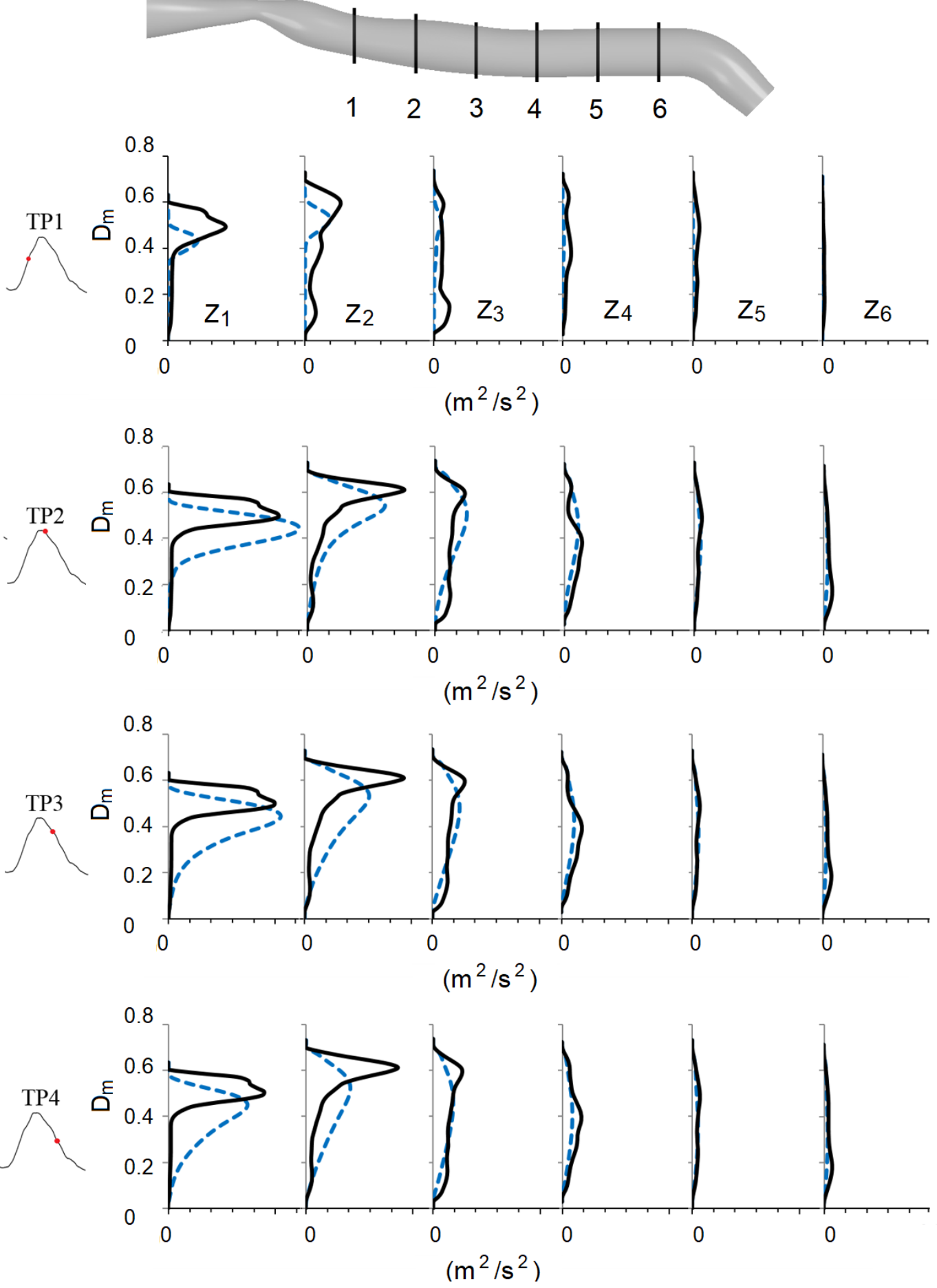

Fig. 8. Turbulent kinetic energy (TKE) profiles at several locations $\left(Z_{1}\right.$ to $Z_{6}$ ) in the ICA at different time points (TP1 - TP4). Dashed blue curve shows SST-Tran and black continuous curve shows LES results. No PIV data were available for comparison. 


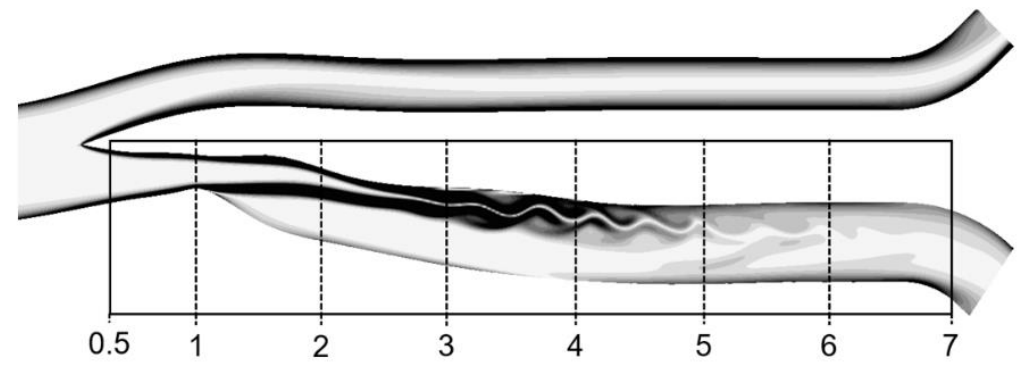

(a)

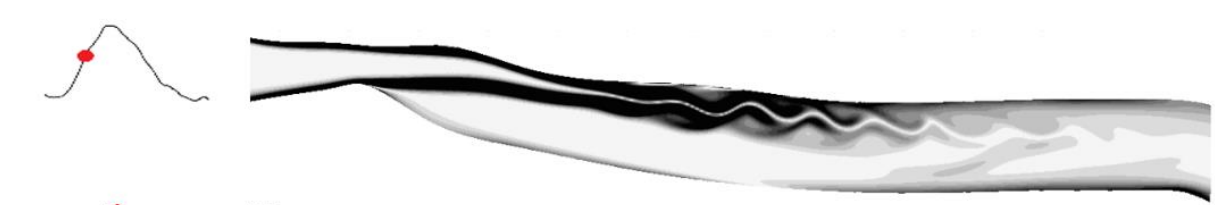

(b)

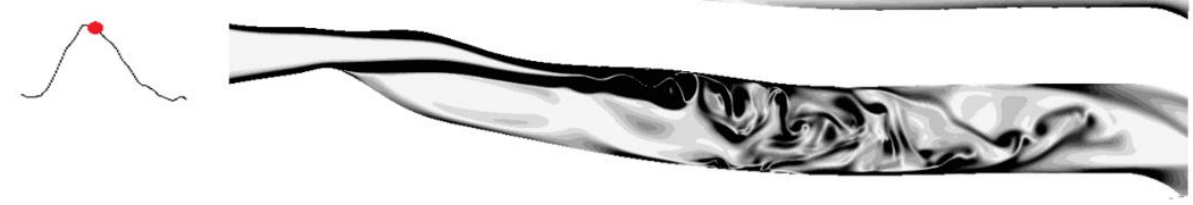

(c)

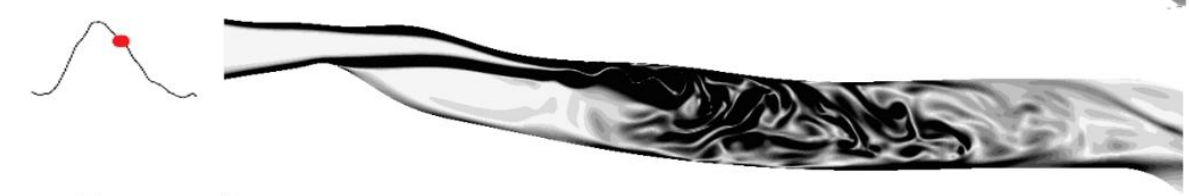

(d)

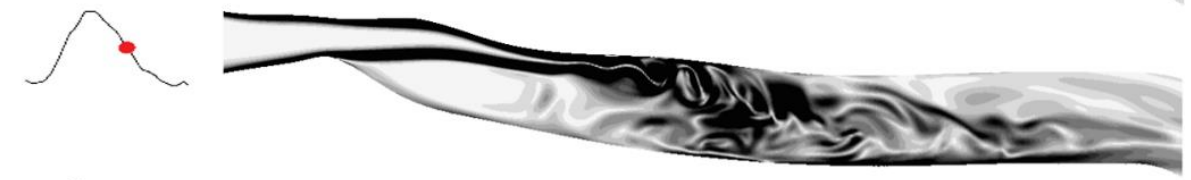

(e)
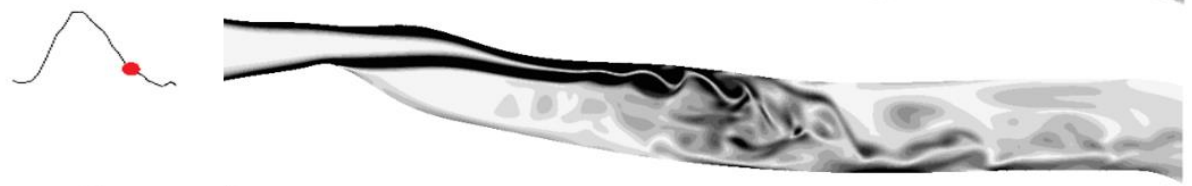

(f)

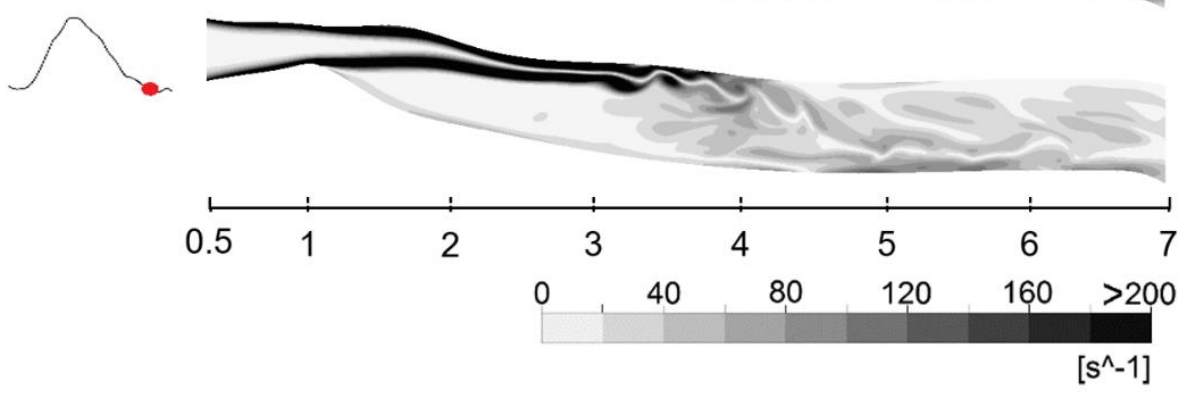

Fig. 9. Vorticity magnitude contours extracted from LES results in the post-stenotic region of ICA at different time-points (a)-(f). Contours shown are in $\mathrm{x}$-y plane. 


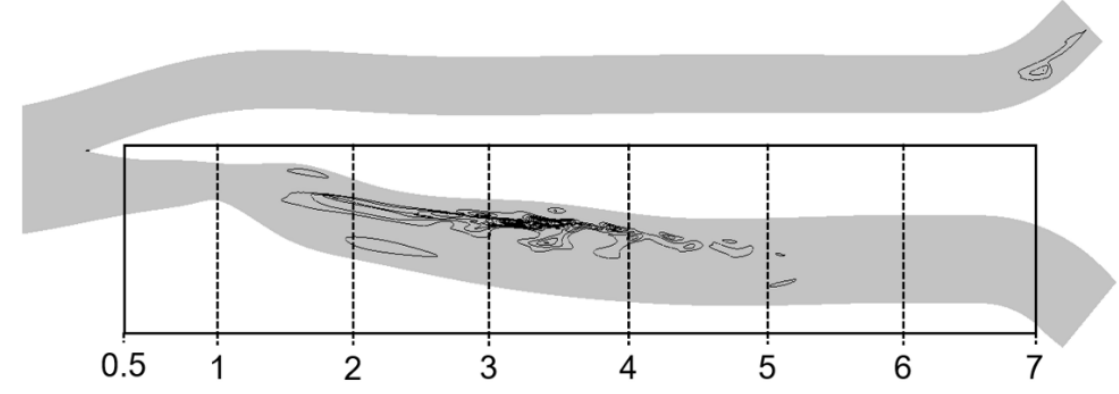

(a)
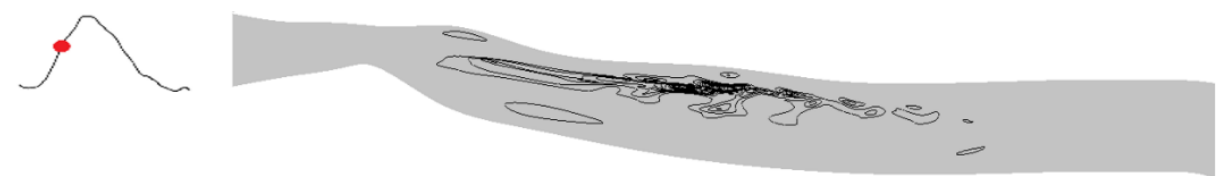

(b)
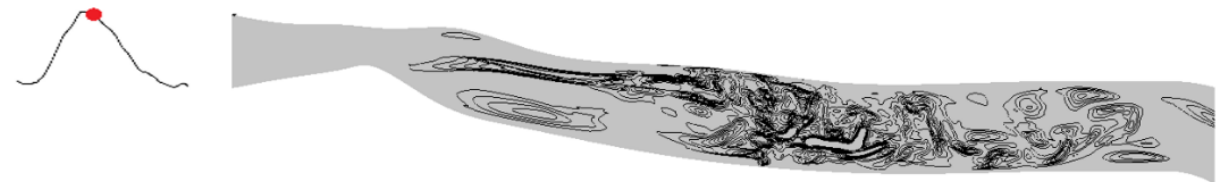

(c)
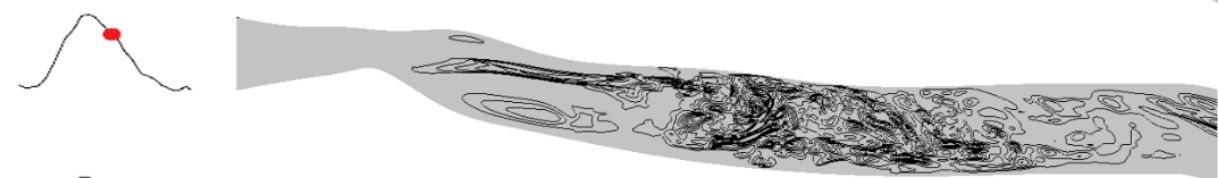

(d)
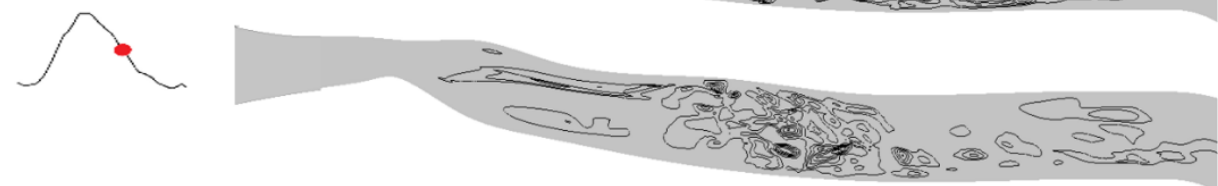

(e)
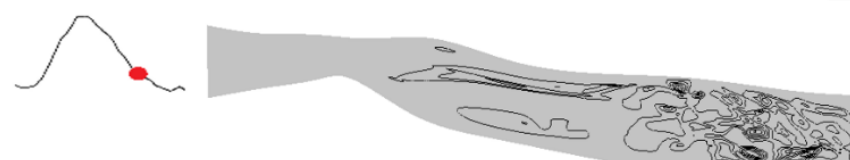

(f)

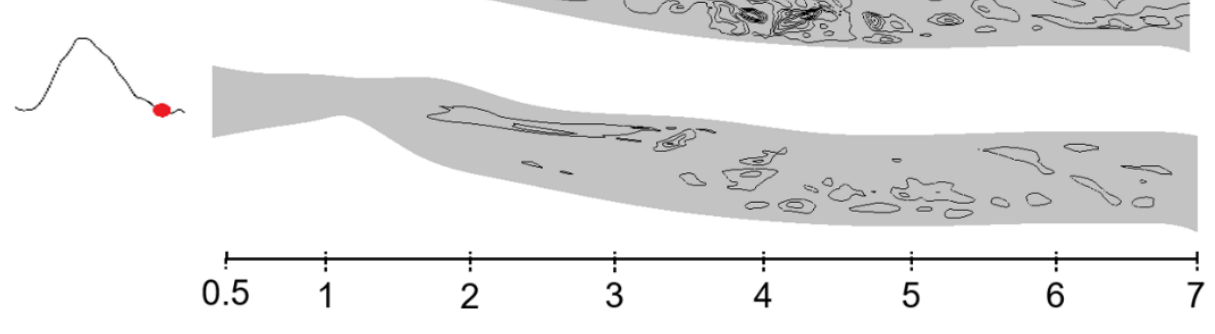

Fig. 10. Normalized eddy viscosity $\left(v_{t} / v\right)$ contours extracted from LES result in the $x$-y plane of the post-stenotic region of ICA at different timepoints (a)-(f). Contours are in the range of 0-0.10. 